\section{TÜV SÜD-Studie zur Bedeutung von Datenschutz im Mittelstand}

Für Verbraucher ist Datenschutz ein zentrales Thema. Doch ist Unternehmen auch ein wirksamer Schutz der personenbezogenen Daten wichtig? Um dieser Frage auf den Grund zu gehen, hat TÜV SÜD in Zusammenarbeit mit der Ludwig-Maximilians-Universität (LMU) München die Studie "Datenschutz 2012" durchgeführt. Wie bei der vorangegangenen Studie im Jahr 2011 wurden überwiegend mittelständische Unternehmen zu ihrer Einstellung zum Schutz personen-bezogener Daten, zu ihrem Informationsstand und zur tatsächlichen Umsetzung befragt. Für einen Großteil der Teilnehmer nimmt der Datenschutz zwar einen hohen Stellenwert ein, dennoch ist hier ein leichter Rückgang gegenüber dem Vorjahr festzustellen.

Unter der Schirmherrschaft des Bayerischen Landesamtes für Datenschutzaufsicht (BayLDA) realisierte die TÜV SÜD Management Service GmbH gemeinsam mit der Fakultät für Psychologie und Pädagogik der LMU München die Studie mit dem Titel „Datenschutz 2012“. „Die Studie zeigt ein gutes Bild über die Verankerung des Datenschutzes in den Unternehmen und leider auch, dass noch einige Luft nach oben ist. Anlass für Unternehmen und Aufsichtsbehörden, sich entspannt zurückzulehnen, besteht noch lange nicht", äußert sich dazu Thomas Kranig, Präsident des BayLDA. Ein Großteil der von März bis Juli 2012 befragten Unternehmen sind wie bereits im Vorjahr Mittelständler mit bis zu 499 Mitarbeitern. 60 Prozent aller Teilnehmer kommen dabei aus den Bundesländern Bayern, Nordrhein-Westfalen und Baden-Württemberg. Die Studie stellte Fragen nach dem Stellenwert des Datenschutzes in Unternehmen und deren Selbsteinschätzung dazu. Außerdem wurden der Umgang von Mitarbeiter, Kunden und Partner mit Datenschutzthemen und Management des Datenschutzes sowie Qualität des Datenschutzes eruiert. Allgemein lässt sich festhalten, dass die Kernergebnisse aus der Studie des vergangenen Jahres bestätigt werden. So ist der Stellenwert des Themas Datenschutz bei den Befragten zwar leicht zurückgegangen, dennoch ist er für knapp zwei Drittel (58 Prozent) noch immer hoch bis sehr hoch.

Defizite bei der Information von Kunden und bei Verpflichtung von Partnern

Dagegen gibt es im Bereich „Kunden und Datenschutz" wie im Vorjahr weiterhin Potenzial für Verbesserungen. Bei knapp einem Viertel der Unternehmen werden Kunden noch immer nicht über die mögliche Verwendung ihrer Daten informiert, was gegen geltendes Recht verstößt. Der größte Handlungsdarf ergibt sich gemäß der Studie im Bereich "Partner und Datenschutz". Eine regelmäßige Überprüfung von Partnern, die mit personenbezogenen Daten des Unternehmens in Berührung kommen wie beispielsweise externe Service-Center, wird in fast 60 Prozent der Fälle nicht vorgenommen. Mangelhafter Datenschutz des Partners kann jedoch leicht auf das eigene Unternehmen zurückfallen.

\section{Datenschutz geht nicht nebenbei}

„Der Schutz personenbezogener Daten ist ein so wichtiges Thema, dass es nicht nebenbei erledigt werden kann, sondern ein gutes Datenschutzmanagement erfordert", erklärt Rainer Seidlitz, Leiter IT- Security bei der TÜV SÜD Management Service GmbH. Dadurch verändert sich auch die Rolle des Datenschutzbeauftragten. Die schriftliche Bestellung einer für diese Rolle fachlich und persönlich geeigneten Person erfüllt zwar die entsprechende gesetzliche
Vorschrift, aber noch längst nicht alle Anforderungen für den wirksamen Schutz personenbezogener Daten. Um ein gutes Schutzniveau zu gewährleisten, sollte der Datenschutzbeauftragte daher die Rolle eines Auditors und Datenschutz-Managers übernehmen. Dafür braucht er jedoch einen unmittelbaren Zugang zum Management und dessen Unterstützung, fundiertes Wissen über die Managementsysteme des Unternehmens und eine entsprechend umfassende fachliche Ausbildung.

Hat ein Unternehmen nicht die nötigen Ressourcen oder das richtige Know-how für einen professionellen Datenschutz, sollte es in Betracht ziehen, das Angebot eines externen Dienstleisters zu nutzen. Dieser stellt sowohl das Personal als auch das Wissen zur Verfügung, um die Anforderungen an den Datenschutz zu erfüllen, was wiederum die Grundlage für einen hohen Vertrauensfaktor bei bestehenden und potenziellen Kunden ist. TÜV SÜD hat im Bereich Datenschutz bereits seit vielen Jahren Erfahrungen gesammelt und bietet als Kompetenzzentrum Beratungen, Dienstleistungen und Prüfungen rund um das Thema Datenschutz.

Mehr Informationen unter www.tuev-sued.de/...

\section{„Ersetzendes Scannen“: TeleTrusT- Informationstag zur BSI-Richtlinie am 19.04.2013 in Berlin}

Nahezu jedes Unternehmen und jede Organisation stand bereits vor der Herausforderung des rechtssicheren „Ersetzenden Scannens". In vielen Fällen kommt gescannten Dokumenten nicht die gleiche gesetzliche Beweiskraft zu wie einem Original-Papierbeleg. Das Bundesamt für Sicherheit in der Informationstechnik (BSI) hat sich dieser Problematik angenommen und die neue BSI-Richtlinie TR RESISCAN 03138 entwickelt. Ziel der Richtlinie ist es, Anwendern und Dienstleistern höhere Rechtssicherheit beim „Ersetzenden Scannen“ zu verschaffen. Anfang 2013 ist die Veröffentlichung der Richtlinie im Bundesanzeiger geplant. Um Anwendern und Dienstleistern eine Hilfestellung bei der Umsetzung zu geben, veranstaltet TeleTrusT in Kooperation mit dem BSI am 19.04.2013 in Berlin einen gemeinsamen Informationstag.

Unternehmen und Organisationen erhalten wichtige praktische Hinweise sowie rechtliche Hintergrundinformationen, ergänzt um Erfahrungsberichte von Unternehmen. Themen des Informationstages sind u.a.:

- Richtlinie BSI TR RESISCAN 03138 - Eine Einführung;

- Schutzbedarfsanalyse, Bedrohungsanalyse, Risikoanalyse;

- Umsetzung der Richtlinie - Modularer Anforderungskatalog;

- Beweiswerterhaltende Langzeitspeicherung gemäß BSI-Richtlinie TR ESOR 03125 - nächster logischer Schritt nach dem ersetzenden Scannen;

- Aufbewahrungspflichten und Beweiswert gescannter Urkunden:

- RESISCAN durch Dritte - Rechtliche Anforderungen an die Beauftragung;

- Ersetzendes Scannen und praktische Umsetzung der BSI TR RESISCAN dargestellt am Beispiel einer Behörde und eines Krankenhauses.

Programm und Anmeldeformular unter http://www.teletrust.de/veranstaltungen/ersetzendes-scannen/ 